\title{
Cytotoxic and Antiviral Properties of Novel Dehydroabietylamine Salts
}

Kseniya S Kovaleva ${ }^{1,2}$, Alena A Kononova ${ }^{2}$, Vlad A Korobeynikov ${ }^{2}$, Sergey V Cheresiz ${ }^{2,3}$, Vladimir V Zarubaev ${ }^{4}$, Anna A Shtro ${ }^{4}$, Yana R Orshanskaya ${ }^{4}$, Olga I Yarovaya ${ }^{1,2 *}$, Andrey G Pokrovsky ${ }^{2}$ and Nariman F Salakhutdinov $v^{1,2}$

${ }^{1}$ NN Vorozhtsov Novosibirsk Institute of Organic Chemistry, Siberian Branch, Russian Academy of Sciences, Lavrentjev Avenue 9, 630090, Novosibirsk, Russia ${ }^{2}$ Novosibirsk State University, Pirogova, Novosibirsk, Russia

${ }^{3}$ Institute of Internal and Prevention Medicine, Siberian Branch of Russian Academy of Medical Sciences, Bogatkova, Novosibirsk, Russia

${ }^{4}$ Department of Chemotherapy, Influenza Research Institute, Petersburg, Russia

\begin{abstract}
Here, we report the synthesis of a series of dehydroabietylamine (DAA) salts. We studied the cytotoxic activity of DAA salts and their and antiviral properties against influenza virus $A(H 1 N 1) p d m 09$ We further compared these parameters to those of DAA itself and to organic acids used as counterions. We observed that the DAA, its hydrochloride $(\mathrm{DAA} \cdot \mathrm{HCl})$ and organic salts had similar cytotoxicity profiles in three cancer cell lines: MDA-MB-231, MCF-7, U-87 MG. Meanwhile the cytotoxicity of same organic salts of DAA with respect to melanoma cell line SK-Mel-8 was higher than those DAA and DAA.HCl: CTD50 values of 5 from 14 synthesized salts were 2-2.8 times lower than that of CTD50 for DAA. $\mathrm{HCl}$. Pure organic acids used for the synthesis of DAA salts were noncytotoxic to cancer cells, except ursolic and glycyrrhetic acid having moderate cytotoxicity. Further, we found that modification of DAA with counterions derived from the selected organic acids does not affect, in most cases, the DAA antiviral activity (except compound $4 \mathrm{n}$, DAA glycyrrhetate). The toxicity of this compound was significantly lower than that of its constituting ions, thereby making the selectivity index of this compound against the $A(H 1 N 1)$ pdm09 virus several times higher than that of other DAA salts.
\end{abstract}

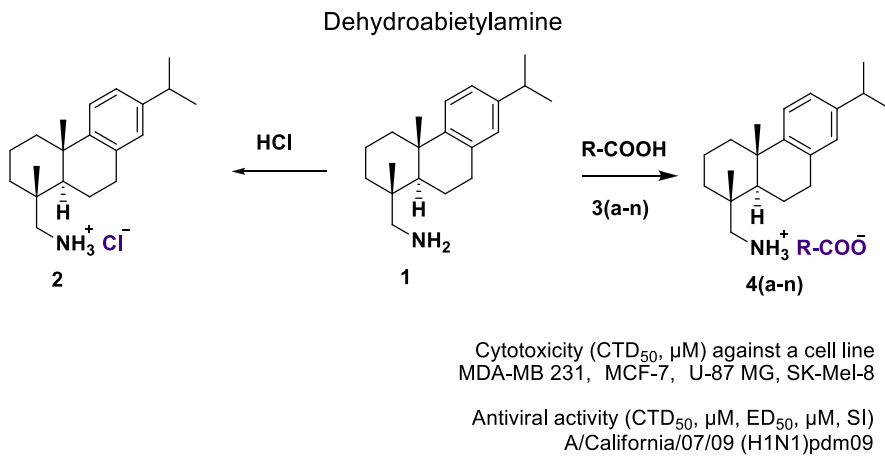

Keywords: Dehydroabietylamine; Counterion; Pharmaceutical salt (ionic derivative); Anti-cancer agent; Influenza virus; Anti-viral activity

\section{Dehydroabietylamine Salts}

Natural compounds are an important constituent of many drugs, with derivatives thereof used extensively in oncology. Approximately $60 \%$ of clinically approved antitumour drugs are synthesized on the basis of secondary metabolites [1]. One such example, dehydroabietylamine (DAA, also referred to in the literature as leelamine) is an abietane diterpenic amine, manufactured industrially from gum resin through the nitrile of dehydroabietic acid (DHA). DHA is present in gum resins of coniferous plants belonging to the genera Pinus, Picea, Abies, and Larix. A particularly high level of DHA (71\%) occurs in the gum resin of the Picea obovata spruce [2]. Indeed, the total world production of this natural tricyclic diterpenoid acid is approximately 1.2 million tons per year.

A number of studies have shown that DAA and its derivatives exhibit a wide range of biological activities: DAA derivatives containing the primary and secondary amino groups demonstrate antiulcer activity [3] and substituted Schiff bases derived from DAA and benzaldehyde derivatives exhibit bactericidal activity against Staphylococcus aureus, Bacillus subtilus, and Escherichia coli [4]. The cytotoxic properties of a wide range of amino group derivatives have been studied in HepG2 liver carcinoma cells [5]; further, an adduct of DAA and quinine was found to exhibit a high antitumour activity on prostate carcinoma cells EJ and PC-3, whilst exhibiting a low toxicity on normal cells [6]. Studies have also been performed on the cytotoxic effect of amides containing DAA and piperazine or polyamine cycles in HepG2 liver carcinoma and MCF-7 breast cancer cells [7]; on the antibacterial and antioxidant activities of DAA derivatives containing a heterocyclic moiety [8]; on Schiff bases derived from DAA with a modified aromatic ring as anticancer agents in HepG2 and L02 cell lines [9] and on the antimalarial activity and low toxicity of $\mathrm{N}$-dehydroabietylbenzamides [10]. Finally, the cytostatic properties of dehydroabietylamine hydrochloride (DAA.HCl) have been studied in a number of cancer

*Corresponding author: Olga I Yarovaya, NN Vorozhtsov Novosibirsk Institute of Organic Chemistry, Siberian Branch, Russian Academy of Sciences, Lavrentjev Avenue 9, 630090, Novosibirsk, Russia, Tel: +73833308870; E-mail: ooo@nioch.nsc.ru

Received October 13, 2016; Accepted October 21, 2016; Published October 25, 2016

Citation: Kovaleva KS, Kononova AA, Korobeynikov VA, Cheresiz SV, Zarubaev VV, et al. (2016) Cytotoxic and Antiviral Properties of Novel Dehydroabietylamine Salts. Med Chem (Los Angeles) 6: 642-646. doi:10.4172/2161-0444.1000408

Copyright: (c) 2016 Kovaleva KS, et al. This is an open-access article distributed under the terms of the Creative Commons Attribution License, which permits unrestricted use, distribution, and reproduction in any medium, provided the original author and source are credited. 
cells [11]. Recently, special attention has been paid to the mechanism of dehydroabietylamine hydrochloride action with the finding that DAA is an inhibitor that simultaneously suppresses several cellular signaling pathways (PI3K, MAPK, and STAT3) associated with malignant transformation of the cell, particularly, in melanoma [12,13]. As the first drug of this type, DAA. $\mathrm{HCl}$ has a unique capability to retain the activity against recurrent resistant melanoma, a malignancy, the cells of which become resistant to the modern chemotherapeutic drugs targeting melanoma and able to inhibit only a single signaling pathway. It is a lysosomotropic drug with a mechanism of action associated with disruption of intracellular cholesterol transport and receptor-mediated endocytosis, leading to the shutdown of receptor tyrosine kinase (RTK) and inhibition of signaling pathways PI3K, MAPK, and STAT3. This, in turn, causes suppression of melanoma cell proliferation, an increase in the level of malignant cell apoptosis, and a decrease in tumor vascularization and, most importantly, preserves the efficacy of the DAA anticancer effect against recurrent resistant melanoma cells resistant to modern antimelanoma drugs (Zelboraf). DAA has been shown to have a high inhibitory efficacy for selective destruction of melanoma cells by reducing the cell proliferation level and increasing apoptosis [14]. Recently successfully studied the antiviral activity of ferruginol and some analogues synthesized from dehydroabietylamine against HHV-1, HHV-2 and DENV-2 [15].

Despite the importance of the obtained data on the biological properties of dehydroabietylamine and dehydroabietylamine hydrochloride, salts of this amine with other acids have not previously been produced and studied. Interest in producing ammonium salts of organic compounds results from observations of high biological activity in various representatives of this class of compounds [16]. Conversion of a biologically active compound into an ionic form leads to changes in physico-chemical properties, primarily in the solubility of the derivative [17]. Some drugs are known to be water-soluble "pharmaceutical salts" consisting of a pharmacologically active ion and a pharmaceutically appropriate counterion [18]. This approach to studying the biological activity of other conjugates is also used in the field of medicinal chemistry. For example, several studies investigated ionic betulin derivatives and their cytotoxic properties in various cancer cell lines [19], as well as their antiviral activity [20]. The aim of the work presented here was to produce a number of dehydroabietylamine salts and investigate their biological properties, in particular, their cytotoxic and antiviral activity.

The cytotoxicity of all synthesized compounds was evaluated using the methyl tetrazolium assay (MTT) in several cancer cell lines, including MCF-7 and MDA-MB-231 mammary gland adenocarcinoma cells, U-87 MG glioblastoma cells, and SK-Mel-8 melanoma cells as well as the MDCK non-transformed canine cell line. MCF-7 and MDA-MB-231 cell lines are the most widely used for studying mammary tumors. Morphologically, MCF-7 cells belong to the luminal subtype A and are hormone-sensitive due to expression of oestrogen and progesterone receptors. MDA-MB-231 line cells possess properties of the basal epithelium and are considered to undergo the epithelial-mesenchymal transformation due to vimentin expression. Accordingly, this line is used as a model of aggressive lowdifferentiated mammary gland adenocarcinoma with a high metastatic potential. U-87 MG is an epithelial morphology cell culture that is derived from a human malignant glioblastoma and routinely used as a glioma model. SK-Mel-8 is a primary skin melanoma-derived cell line used as a melanoma model. MDCK is the canine kidney epithelial cell line used in influenza research.

Cells were cultivated in D-MEM (MCF-7, MDA-MB-231, and
SK-Mel-8), alpha-MEM (U-87 MG), or MEM growth media supplied with $10 \%$ bovine serum, L-glutamine, and penicillin/streptomycin at $37^{\circ} \mathrm{C}$ in $5 \% \mathrm{CO}_{2}$. We prepared a set of DAA salts; the structures of the original amine and acids used are shown in Scheme 1. As counterions, we used an aliphatic carboxylic valproic acid (3a), cinnamic acid (3b), and its natural analogues: caffeic (3c) and ferulic (3d) acids; aromatic acids (3e, 3f), sulfoaromatic acid (3g), and heteroaromatic acids (3h, $3 \mathbf{i})$. In addition, we obtained DAA salts of natural acids: DAA (3j), deoxycholic acid (3k), betulonic acid (3l), ursolic acid (3m) and glycyrrhetic acid (3n). The natural acids used have a broad spectrum of biological activities. Indeed, the antifungal [21], antiherpetic [22], and anti-inflammatory [23] activities of DHA (3j) have previously been demonstrated. Deoxycholic acid is a bile acid representative of an immunostimulant of innate immunity/immune system and is involved in the inflammation recovery and wound healing [24], as well as in maintaining homeostasis of lipids, glucose, and cholesterol. In addition, bile acids are recognized to play an important role in the promotion of colon and oesophageal cancers [25]. Ursolic acid is a pentacyclic triterpenoid widely found in various medicinal herbs and several foods [26], thus constituting an integral part of the human diet. It possesses many biological effects, including antitumour, anti-inflammatory, and antioxidant activities [27].

All salts were purified by recrystallization from an appropriate solvent, followed by measurement of individual melting points. IR spectra of salts (4a-n) were observed to lack the free hydroxyl group line present in spectra of the free acids, indicating the formation of a corresponding salt.

We studied the cytotoxicities of the original amine and synthesized dehydroabietylamine salts $(\mathbf{2}, \mathbf{4 a}-\mathbf{n})$ in several cancer cell lines, including two mammary carcinoma cell lines (MDAMB-231 and MCF-7), the U-87 MG glioblastoma and SK-Mel-8 melanoma cell lines. As seen from the $\mathrm{CTD}_{50}$ values in Table 1, the series of studied compounds have a similar toxicity profiles in three cancer cell lines: MDA-MB-231, MCF-7, U-87 MG. Meanwhile the cytotoxicity of same aromatic salts of DAA with respect to melanoma cell line SK-Mel-8 was higher than those DAA and DAA.HCl. Thus, $\mathrm{CTD}_{50}$ values of $\mathbf{4 b}, \mathbf{4 e}, \mathbf{4 f}$, 4g, $4 \mathbf{h}$ and $4 \mathbf{i}$ were 2-2.8 times lower than that of $\mathrm{CTD}_{50}$ for DAA.HCl.
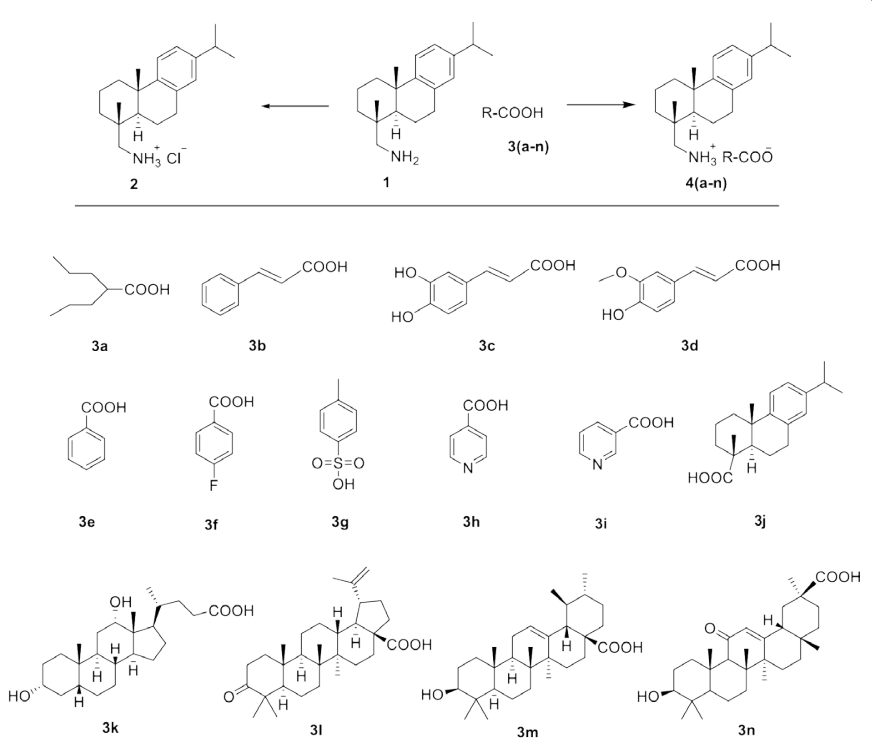

Scheme 1: The structures of the original amine and acids used. 
Citation: Kovaleva KS, Kononova AA, Korobeynikov VA, Cheresiz SV, Zarubaev VV, et al. (2016) Cytotoxic and Antiviral Properties of Novel Dehydroabietylamine Salts. Med Chem (Los Angeles) 6: 642-646. doi:10.4172/2161-0444.1000408

The greatest activity showed the dehydroabietylamine isonicotinate $\mathbf{4 f}$ $\left(\mathrm{CTD}_{50} 2.7 \mu \mathrm{M}\right)$. This fact can be considered as very important, because the most promising is the study of dehydroabietylamine derivatives against melanoma cells.

With regard to other cell lines such as MDA-MB-231, MCF-7, U-87 MG, the most active synthesized DAA salts had cytotoxic activities close to those of DAA or its hydrochloride, while substitution of the chloride with an organic acid counterion reduced the DAA cytotoxicity in other cases. The maximum difference in $\mathrm{CD}_{50}$ between the most and least toxic DAA salts was only $\sim 3.5$ times for compounds $\mathbf{4 h}$ and $\mathbf{4 j}$ in the MDA-MB-231 cell line. In general, cells of different lines showed slightly different sensitivity to DAA salts, with U-87 MG cells being most resistant (except compounds $\mathbf{4} \mathbf{i}$ and $\mathbf{4 c}$ that appeared to be less toxic in MCF-7 cells).

Meanwhile, the organic acids used as counterions for the studied DAA salt series showed no detectable cytotoxicity in the range of concentrations from 200 to $800 \mu \mathrm{M}$, except ursolic and glycyrrhetic acid having moderate cytotoxicity.

The antiviral activity of the synthesized compounds was evaluated against the epidemiologically important strain of the influenza A virus A/California/07/09 (H1N1)pdm09. The findings are summarized in Table 2.

The acids selected as counterions, exhibited no noticeable cytotoxic activity at concentrations of up to $500 \mu \mathrm{M}$. The $50 \%$ cytotoxic concentration of dehydroabietylamine chloride (2) was $6.8 \mu \mathrm{M}$. In some cases, substitution of the chlorine with other counterions led to a decrease in the compound toxicity. For example, the cytotoxic concentration increased 6- (4a), 4- (4g), 12- (4k), 4- (4m), and 119-fold (4n) when aliphatic dicarboxylic, sulfoaromatic, betulonic, ursolic, and glycyrrhetic acids, respectively, were used as counterions.

In most cases, modification of dehydroabietylamine with organic acid counterions did not lead to an increase in the antiviral activity. In contrast, if an organic acid had this activity, a combination of the acid with dehydroabietylamine led to the loss of antiviral properties (selectivity index reduction) in the resulting salt. Indeed, the selectivity index of caffeic acid decreased from 9 for free acid to 1 for its dehydroabietylamine salt ( $3 c$ and $\mathbf{4 c}$, respectively) due to an increase in cellular toxicity. The same was observed for the pair of (3j) and (4j).

Of particular interest is the modification of dehydroabietylamine with the glycyrrhetic acid counterion. The toxicity of the resulting salt was much lower than that of each of the ions separately, while the selectivity index towards the influenza virus increased from 1 to 35 (compounds 2, 3n, and $\mathbf{4 n}$ ). These properties of triterpene salts of dehydroabietylamine warrant further investigation.

In this study, we have demonstrated that the biological activity of dehydroabietylamine salts can be affected by the type of organic acid used as counterion. We observed that the DAA, its hydrochloride (DAA $\cdot \mathrm{HCl}$ ) and organic salts had similar cytotoxicity profiles in three cancer cell lines: MDA-MB-231, MCF-7, U-87 MG. The cytotoxicity of same organic salts of DAA with respect to melanoma cell line SK-Mel-8 was higher than those DAA and DAA. $\mathrm{HCl}$. Pure organic acids used for the synthesis of DAA salts were non-cytotoxic to cancer cells, except ursolic and glycyrrhetic acid having moderate cytotoxicity. In general, combination of dehydroabietylamine with organic acids resulted in decrease of anti-viral properties due to high cell toxicity of the complex salt. Nevertheless, further, we have shown that the use of natural acids (betulonic and glycyrrhetic) as a counterion greatly reduced the toxicity of the resulting salts, and that these counterions had no appreciable cytotoxic activity at concentrations of up to $2000 \mu \mathrm{M}$.

\section{Acknowledgments}

This work was partially supported by the Russian Foundation Research 5-123 00017 and by Russian Ministry of Education and Science "Organization of scientific research 151".

\section{Supplementary Data}

Supplementary data (experimental procedures for the synthesis of compounds $a-4 n$. IR spectra and melting points of compounds $\left.{ }^{45} 4 a-4 n\right)$. The experimental protocol to compounds $4 \mathrm{a}-4 \mathrm{n}$ and their analytical characterization are reported in the Supplementary Information. The experimental protocol to toxicity study of compounds $4 \mathrm{a}-4 \mathrm{n}$ is reported in the Supplementary Information. The experimental protocol to antiviral study of compounds $4 a-4 n$ is reported in the Supplementary Information.

\begin{tabular}{|c|c|c|c|c|c|}
\hline & & \multicolumn{4}{|c|}{ Cytotoxicity $\left(\mathrm{CTD}_{50} . \mu \mathrm{M}\right)$ against a cell line } \\
\hline \multicolumn{2}{|c|}{ Compound } & MDA-MB 231 & MCF-7 & U-87 MG & SK-Mel-8 \\
\hline \multicolumn{2}{|c|}{1} & $16.3 \pm 0.2$ & $14.5 \pm 1.0$ & $14.0 \pm 1.3$ & $10.9 \pm 0.3$ \\
\hline \multicolumn{2}{|c|}{2} & $11.2 \pm 0.6$ & $16.2 \pm 1.3$ & $15.1 \pm 0.7$ & $7.5 \pm 1.2$ \\
\hline \multirow{2}{*}{ a } & 3 & NT & NT & NT & $>300$ \\
\hline & 4 & NT & NT & NT & $16.5 \pm 1.3$ \\
\hline \multirow{2}{*}{ b } & 3 & $>675$ & $>675$ & $>675$ & $>300$ \\
\hline & 4 & $11.3 \pm 0.5$ & $27.3 \pm 1.1$ & $19.1 \pm 0.5$ & $3.6 \pm 0.2$ \\
\hline \multirow{2}{*}{ c } & 3 & $>555$ & $>555$ & $>555$ & $>300$ \\
\hline & 4 & $15.3 \pm 0.6$ & $24.7 \pm 1.2$ & $21.5 \pm 0.8$ & $21.5 \pm 1.5$ \\
\hline \multirow{2}{*}{ d } & 3 & $>515$ & $>515$ & $>515$ & $>300$ \\
\hline & 4 & $20.0 \pm 0.1$ & $16.3 \pm 1.3$ & $20.7 \pm 0.5$ & $32.3 \pm 0.9$ \\
\hline \multirow{2}{*}{ e } & 3 & $>820$ & $>820$ & $>820$ & $>300$ \\
\hline & 4 & $11.3 \pm 1.8$ & $22.2 \pm 0.5$ & $20.2 \pm 1.0$ & $3.3 \pm 0.4$ \\
\hline \multirow{2}{*}{$\mathbf{f}$} & 3 & $>710$ & $>710$ & $>710$ & $>300$ \\
\hline & 4 & $12.1 \pm 0.3$ & $14.2 \pm 0.8$ & $19.1 \pm 0.1$ & $3.7 \pm 1.4$ \\
\hline \multirow{2}{*}{ g } & 3 & $>580$ & $>580$ & $>680$ & $>300$ \\
\hline & 4 & $10.6 \pm 1.1$ & $18.1 \pm 1.5$ & $19.3 \pm 0.9$ & $4.0 \pm 1.1$ \\
\hline \multirow{2}{*}{ h } & 3 & $>810$ & $>810$ & $>810$ & $>300$ \\
\hline & 4 & $10.4 \pm 1.3$ & $19.5 \pm 0.8$ & $20.0 \pm 1.0$ & $2.7 \pm 0.3$ \\
\hline \multirow{2}{*}{$\mathbf{i}$} & 3 & $>810$ & $>810$ & $>810$ & $>300$ \\
\hline & 4 & $14.2 \pm 0.4$ & $14.0 \pm 0.6$ & $23.4 \pm 0.6$ & $3.5 \pm 0.2$ \\
\hline
\end{tabular}


Citation: Kovaleva KS, Kononova AA, Korobeynikov VA, Cheresiz SV, Zarubaev VV, et al. (2016) Cytotoxic and Antiviral Properties of Novel Dehydroabietylamine Salts. Med Chem (Los Angeles) 6: 642-646. doi:10.4172/2161-0444.1000408

\begin{tabular}{|c|c|c|c|c|c|}
\hline \multirow{2}{*}{$\mathbf{j}$} & 3 & $250 \pm 30$ & $240 \pm 30$ & $>300$ & $>300$ \\
\hline & 4 & $34.4 \pm 2.4$ & $35.2 \pm 1.5$ & $37.3 \pm 1.0$ & $30.0 \pm 2.7$ \\
\hline \multirow{2}{*}{ k } & 3 & $>255$ & $>255$ & $>255$ & $>300$ \\
\hline & 4 & $16.7 \pm 0.6$ & $14.3 \pm 0.4$ & $25.1 \pm 1.0$ & $19.2 \pm 1.6$ \\
\hline \multirow{2}{*}{ I } & 3 & $>220$ & $150 \pm 40$ & $>220$ & $66.9 \pm 5.8$ \\
\hline & 4 & $29.4 \pm 1.3$ & $29.0 \pm 2.5$ & $29.2 \pm 1.0$ & $20.0 \pm 4.1$ \\
\hline \multirow{2}{*}{ m } & 3 & $87.6 \pm 0.9$ & $71 \pm 2$ & $89.8 \pm 2.1$ & $90.3 \pm 4.7$ \\
\hline & 4 & $13.3 \pm 0.8$ & $30.5 \pm 0.7$ & $31.9 \pm 0.5$ & $20.2 \pm 2.2$ \\
\hline \multirow{2}{*}{$\mathrm{n}$} & 3 & $26 \pm 2.5$ & $31 \pm 2.5$ & $>100$ & $>100$ \\
\hline & 4 & $31.0 \pm 3.0$ & $33.7 \pm 0.8$ & $31.9 \pm 1.2$ & $22.0 \pm 3.1$ \\
\hline
\end{tabular}

Table 1: Cytotoxic activity of tested compounds against cancer cell lines.

\begin{tabular}{|c|c|c|c|c|}
\hline \multicolumn{2}{|c|}{ Compound } & $\mathrm{CTD}_{50}, \mu \mathrm{M}$ & $\mathrm{ED}_{50}, \mu \mathrm{M}$ & SI \\
\hline \multicolumn{2}{|c|}{1} & $66.3 \pm 4.1$ & $>35$ & 2 \\
\hline \multicolumn{2}{|c|}{2} & $6.8 \pm 0.3$ & $>9.3$ & 1 \\
\hline \multirow{2}{*}{ a } & 3 & $>2000$ & $>2000$ & 1 \\
\hline & 4 & $27.7 \pm 1.6$ & $23.3 \pm 2.8$ & 1 \\
\hline \multirow{2}{*}{ b } & 3 & $>2000$ & $>2000$ & 1 \\
\hline & 4 & $10.8 \pm 1.0$ & $>6.9$ & 2 \\
\hline \multirow{2}{*}{ c } & 3 & $>1500$ & $183 \pm 21.1$ & 9 \\
\hline & 4 & $4.9 \pm 0.3$ & $>6.5$ & 1 \\
\hline \multirow{2}{*}{ d } & 3 & $>500$ & $>500$ & 1 \\
\hline & 4 & $7.9 \pm 0.5$ & $>6.3$ & 1 \\
\hline \multirow{2}{*}{ e } & 3 & $>2000$ & $>2000$ & 1 \\
\hline & 4 & $7.6 \pm 0.4$ & $3.2 \pm 0.4$ & 2 \\
\hline \multirow{2}{*}{$f$} & 3 & $>2000$ & $>2000$ & 1 \\
\hline & 4 & $15.3 \pm 1.1$ & $12.9 \pm 1.3$ & 1 \\
\hline \multirow{2}{*}{ g } & 3 & $>2000$ & $319.8 \pm 36.2$ & 7 \\
\hline & 4 & $19.9 \pm 1.4$ & $13.1 \pm 1.5$ & 2 \\
\hline \multirow[t]{2}{*}{ h } & 3 & $>2000$ & $>2000$ & 1 \\
\hline & 4 & $4.2 \pm 0.2$ & $4.2 \pm 0.6$ & 1 \\
\hline \multirow{2}{*}{$\mathbf{i}$} & 3 & $>2000$ & $>2000$ & 1 \\
\hline & 4 & $11.3 \pm 0.8$ & $2.4 \pm 0.4$ & 5 \\
\hline \multirow{2}{*}{ j } & 3 & $44.3 \pm 3.3$ & $4.8 \pm 0.5$ & 9 \\
\hline & 4 & $59.9 \pm 4.1$ & $>70$ & 1 \\
\hline \multirow{2}{*}{ k } & 3 & $39.0 \pm 3.3$ & $26.6 \pm 3.0$ & 1 \\
\hline & 4 & $26.4 \pm 1.8$ & $18 \pm 1.9$ & 1 \\
\hline \multirow{2}{*}{1} & 3 & $4.6 \pm 0.4$ & $6.6 \pm 0.5$ & 1 \\
\hline & 4 & $8.2 \pm 0.6$ & $4.9 \pm 0.5$ & 2 \\
\hline \multirow{2}{*}{ m } & 3 & $13.6 \pm 0.9$ & $>6.6$ & 2 \\
\hline & 4 & $13.2 \pm 0.8$ & $3.6 \pm 0.5$ & 4 \\
\hline \multirow{2}{*}{$\mathbf{n}$} & 3 & $74.7 \pm 5.3$ & $>63.8$ & 1 \\
\hline & 4 & $347.0 \pm 22.1$ & $9.8 \pm 1.0$ & 35 \\
\hline
\end{tabular}

${ }^{a} \mathrm{CTD}_{50}$ is the median cytotoxic dose, i.e., the concentration causing $50 \%$ cell death

${ }^{b} \mathrm{ED}{ }_{50}$ is the $50 \%$ effective dose, i.e., the concentration causing $50 \%$ decrease of virus replication

' $\mathrm{SI}$ is the selectivity index, which is the $\mathrm{CTD}_{50} / \mathrm{ED}_{50}$ ratio

Table 2: Activity of tested compounds against the influenza virus $A(\mathrm{H} 1 \mathrm{~N} 1) \mathrm{pdm} 09$.

\section{References}

1. Newman DJ, Cragg GM (2016) Natural Products as Sources of New Drugs from 1981 to 2014. J Nat Prod 79: 629-661.

2. Sandermann WN, Terpentinöl Y (1960) Chemie und Technologie. Berlin/ Göttingen/Heidelberg, Springer, p: 483.

3. Wada H, Kodato S, Kawamori M, Morikawa T, Nakai H, et al. (1985) Antiulcer activity of dehydroabietic acid derivatives. Chem Pharm Bull (Tokyo) 33: 1472-1487.

4. Zhao X, Song DK, Radbil' AB, Radbil' BA (2007) Synthesis and biological activity of Schiff bases derived from dehydroabietylamine and benzaldehyde derivatives. Russian Journal of Applied Chemistry 80: 1373-1375.

5. Lin LY, Bao YL, Chen Y, Sun LG, Yang XG, et al. (2012) N-Benzoyl-12nitrodehydroabietylamine-7-one, a novel dehydroabietylamine derivative induces apoptosis and inhibits proliferation in HepG2 cells. Chem Biol Interact 199: 63-73.
6. Xing Y, Zhang W, Song J, Zhang Y, Jiang X, et al. (2013) Anticancer effects of a novel class rosin-derivatives with different mechanisms. Bioorg Med Chem Lett 23: 3868-3872.

7. Yang X, Qin X, Wang Q, Huang Y (2015) Synthesis and antitumor activities of piperazine-and cyclen-conjugated dehydroabietylamine derivatives. Heterocyclic Communications 21: 233-237.

8. Salehi P, Ayyari M, Bararjanian M, Ebrahimi SN, Aliahmadi A (2014) Synthesis antibacterial and antioxidant activity of novel 2, 3-dihydroquinazolin-4 (1H)-one derivatives of dehydroabietylamine diterpene. Journal of the Iranian Chemical Society 11: 607-613.

9. Liu CX, Lin ZX, Lu Z, Wang YM, Bao YL (2013) Antitumor and scavenging radicals activities of some polyphenols related to dehydroabietylamine derivatives. J Asian Nat Prod Res 15: 819-827.

10. Sadashiva MP, Gowda R, Wu X, Inamdar G, Kuzu OF, et al. (2015) A noncytotoxic $\mathrm{N}$-dehydroabietylamine derivative with potent antimalarial activity. Exp Parasitol 155: 68-73. 
Citation: Kovaleva KS, Kononova AA, Korobeynikov VA, Cheresiz SV, Zarubaev VV, et al. (2016) Cytotoxic and Antiviral Properties of Novel Dehydroabietylamine Salts. Med Chem (Los Angeles) 6: 642-646. doi:10.4172/2161-0444.1000408

11. Gowda R, Madhunapantula SV, Kuzu OF, Sharma A, Robertson GP (2014) Targeting multiple key signaling pathways in melanoma using leelamine. Mol Cancer Ther 13: 1679-1689.

12. Kuzu OF, Gowda R, Sharma A, Robertson GP (2014) Leelamine mediates cancer cell death through inhibition of intracellular cholesterol transport. Mol Cancer Ther 13: 1690-1703.

13. Gowda R, Madhunapantula SV, Kuzu OF, Sharma A, Gavin RP (2013) Natural occurring leelamine inhibits melanoma development by targeting multiple signaling pathways. Cancer Research 73: 5509-5509.

14. Robertson GP, Raghavendragowda CD, Madhunapantula SV, Kuzu OF, Gajanan S (2012) Compositions and methods relating to proliferative diseases. US20120141578 A1.

15. Roa-Linares VC, Brand YM, Agudelo-Gomez LS, Tangarife-Castaño V, Betancur-Galvis LA, et al. (2016) Anti-herpetic and anti-dengue activity of abietane ferruginol analogues synthesized from (+)-dehydroabietylamine. European journal of medicinal chemistry 108: 79-88.

16. Stahl PH, Wermuth CG (2008) Handbook of Pharmaceutical salts properties, selection, and use. John Wiley \& Sons.

17. Qiu Y, Chen Y, Zhang GG, Liu L, Porter W (2009) Developing solid oral dosage forms: pharmaceutical theory and practice.

18. Serajuddin AT (2007) Salt formation to improve drug solubility. Adv Drug Deliv Rev 59: 603-616

19. Suresh C, Zhao H, Gumbs A, Chetty CS, Bose HS (2012) New ionic derivatives of betulinic acid as highly potent anti-cancer agents. Bioorg Med Chem Lett 22 : 1734-1738.
20. Visalli RJ, Ziobrowski H, Badri KR, He JJ, Zhang X, et al. (2015) Ionic derivatives of betulinic acid exhibit antiviral activity against herpes simplex virus type-2 (HSV-2), but not HIV-1 reverse transcriptase. Bioorg Med Chem Lett 25: 3168-3171.

21. González MA, Pérez-Guaita D, Correa-Royero J, Zapata B, Agudelo L, et al (2010) Synthesis and biological evaluation of dehydroabietic acid derivatives Eur J Med Chem 45: 811-816.

22. Tagat JR, Nazareno DV, Puar MS, McCombie SW, Ganguly AK (1994) Synthesis and anti-herpes activity of some a-ring functionalized dehydroabietane derivatives. Bioorganic \& Medicinal Chemistry Letters 4: 1101-1104.

23. Kang MS, Hirai S, Goto T, Kuroyanagi K, Lee JY, et al. (2008) Dehydroabietic acid, a phytochemical, acts as ligand for PPARs in macrophages and adipocytes to regulate inflammation. Biochem Biophys Res Commun 369: 333-338.

24. Vlcek B (1972) Deoxycholic acid as a potential cancerostatic and antivira factor, Advances in Antimicrobial and Antineoplastic Chemotherapy. Urban \& Schwarzenberg, München 2: 145-147.

25. Popadyuk II, Markov AV, Salomatina OV, Logashenko EB, Shernyukov AV, et al. (2015) Synthesis and biological activity of novel deoxycholic acid derivatives. Bioorg Med Chem 23: 5022-5034.

26. Alqahtani A, Hamid K, Kam A, Wong KH, Abdelhak Z, et al. (2013) The pentacyclic triterpenoids in herbal medicines and their pharmacological activities in diabetes and diabetic complications. Curr Med Chem 20: 908-931.

27. Lee SU, Park SJ, Kwak HB, Oh J, Min YK, et al. (2008) Anabolic activity of ursolic acid in bone: Stimulating osteoblast differentiation in vitro and inducing new bone formation in vivo. Pharmacol Res 58: 290-296. 